\title{
Development of Silicon Strip Detector for J-PARC muon g-2/EDM Experiment
}

Yutaro Sato*, Masahiro Ikeno, Tetsuichi Kishishita, Takashi Kohriki, Tatsuya Kume, Tsutomu Mibe, Takeshi Murakami, Naohito Saito, Osamu Sasaki, Nobuhiko Sato, Hiroshi Sendai, Masayoshi Shoji, Toshikazu Takatomi, Manobu Tanaka, Tomohisa Uchida, Kazuki Ueno

High Energy Accelerator Research Organization (KEK), Japan

E-mail: syutaro@post.kek.jp

Hirokazu Ikeda

Japan Aerospace Exploration Agency (JAXA), Japan

Takumi Ito, Kiyotomo Kawagoe, Masato Matama, Shohei Shirabe, Taikan Suehara, Junji Tojo, Yuki Tsutsumi, Takashi Yamanaka, Tamaki Yoshioka

Kyushu University, Japan

Shoichiro Nishimura, Hiromasa Yasuda

The University of Tokyo, Japan

for the J-PARC g-2/EDM experiment (E34)

The muon anomalous magnetic moment $(g-2)_{\mu}$ and electric dipole moment (EDM) are sensitive to new physics beyond the Standard Model. We aim to measure $(g-2)_{\mu}$ with a precision of 0.1 parts per million and search for EDM with a sensitivity of $10^{-21} e \cdot \mathrm{cm}$ at J-PARC. In this experiment, we use a silicon strip detector with high granularity and fast response to detect positrons from muon decay. We report on the development status of the silicon strip detector.

The 39th International Conference on High Energy Physics (ICHEP2018)

4-11 July, 2018

Seoul, Korea

${ }^{*}$ Speaker. 


\section{Introduction}

The muon anomalous magnetic moment $(g-2)_{\mu}$ and electric dipole moment (EDM) are sensitive to new physics beyond the Standard Model (SM). There is a discrepancy between the $(g-2)_{\mu}$ measured by the E821 collaboration at Brookhaven National Laboratory (BNL) [1] and the SM prediction [2]. This difference indicates physics beyond the SM at more than $3 \sigma$ level. To make a definitive conclusion, more precise measurement is desired. The SM predicts the electric dipole moment $(\mathrm{EDM})$ of the muon is very small, beyond our experimental capabilities. If any nonzero value of the EDM is observed, it would imply the existence of new physics.

A new measurement of the $(g-2)_{\mu}$ and the EDM [3] is in preparation at the Japan Proton Accelerator Research Complex (J-PARC) with completely different techniques from the methods used at BNL and Fermi National Accelerator Laboratory [4]. Our goals are to measure the $(g-2)_{\mu}$ with a precision of 0.1 parts per million (ppm), which corresponds to the improvement from the BNL experiment by factor 5 , and to search for the EDM with a sensitivity of $10^{-21} e \cdot \mathrm{cm}$, respectively. We utilize high intensity proton beam at J-PARC and newly developed technique of ultra-cold muon beam, which is produced by thermal muonium productions [5] followed by laser ionization [6] and muon linear acceleration [7]. The ultra-cold muon beam accelerated up to $300 \mathrm{MeV} / \mathrm{c}$ are injected into the storage magnet with a 3 Tesla magnetic field [8]. The positron from muon decay is detected by the silicon strip detector.

\section{Silicon Strip Detector}

To detect circular positron tracks from the $\mu^{+} \rightarrow e^{+} v_{e} \bar{v}_{\mu}$ decay, we locate fast-response silicon strip detectors with high granularity in the storage magnet. The 40 silicon strip vanes are placed radially in the detection volume as shown in Fig. 1 Figure 2 shows the structure of a quarter-vane. Each vane has single-sided p-on-n type silicon strip sensors on both sides with mutually orthogonal strips. Two-dimensional position of a positron track is detected by two layers of the silicon strip sensor.

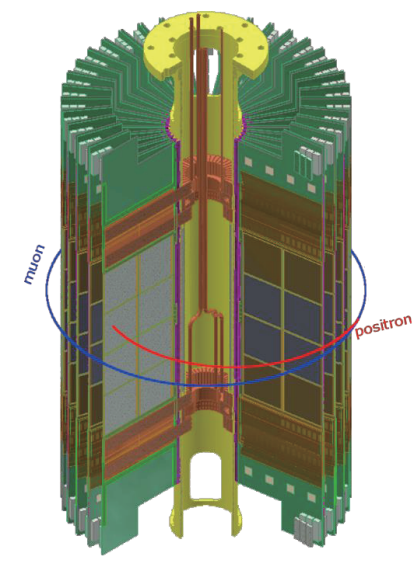

Figure 1: Configuration of the silicon strip detector.

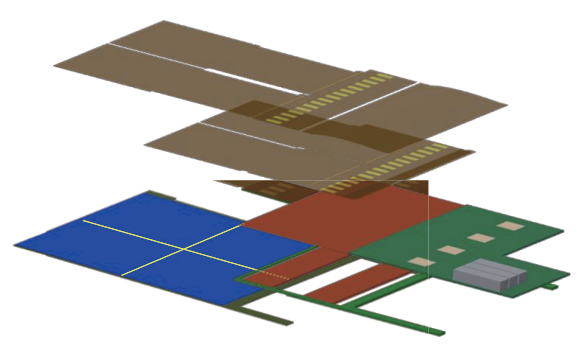

Figure 2: The structure of quarter-vane. 


\section{Development Status}

The silicon strip sensor has been made by Hamamatsu Photonics. Each sensor has 1024 readout strips at a constant strip pitch of $190 \mu \mathrm{m}$. The active area is $97.28 \mathrm{~mm} \times 97.28 \mathrm{~mm}$ with $320 \mu \mathrm{m}$ thickness [9]. The quality assurance method is also being developed : (i) the visual inspection of each sensor, (ii) the measurement of I-V and C-V characterization, and (iii) the strip-by-strip measurement of strip leakage current, strip resistance, coupling capacitance, and inter-strip capacitance by a probe card.

The front-end readout ASIC is required to tolerate a high hit rate of $1.4 \mathrm{MHz}$ per strip, to be stable to the change of hit rate by a factor of $1 / 150$. A full scale prototype readout ASIC "SliT128A" has been fabricated using the Silterra $0.18-\mu \mathrm{m}$ CMOS process. In the prototype, there are 128 readout channels, each of which have an amplifier, a CR-RC shaper, a comparator with a reference voltage set by a 6-bit Digital-to-Analog Converter (DAC), a Time-to-Digital Converter with $5 \mathrm{~ns}$ time sampling, $40.96 \mu$ s buffer memory, and readout blocks. The threshold value at the comparator is adjustable channel by channel using a 6-bit DAC to compensate for channel-to-channel threshold variations. The binary signal from the comparator is sampled with $5 \mathrm{~ns}$ intervals for the period of $40.96 \mu$ s and saved in the memory buffer. The data is read out by a serial communication protocol. Figure 3 shows the count efficiency as a function of threshold at the comparator and equivalent noise charge. The equivalent noise charge is estimated to be 900 electrons with the detector capacitance of $17 \mathrm{pF}$. As a result of the performance test, the SliT128A satisfies the requirements for the muon $g$-2/EDM experiment at J-PARC except for the time-walk and pulse width. In order to improve the performance of time-walk and the pulse width, we have newly designed an analog prototype ASIC "SliT2017TEG". In this prototype ASIC, the differentiator is added at the output of CR-RC shaper. As a result of the evaluation test, the time-walk and the pulse width is considerably improved. The time-walk and pulse width are evaluated to be about $1 \mathrm{~ns}$ and $50 \mathrm{~ns}$, respectively. The design of final version of the front-end readout ASIC "SliT128B" is being finalized.
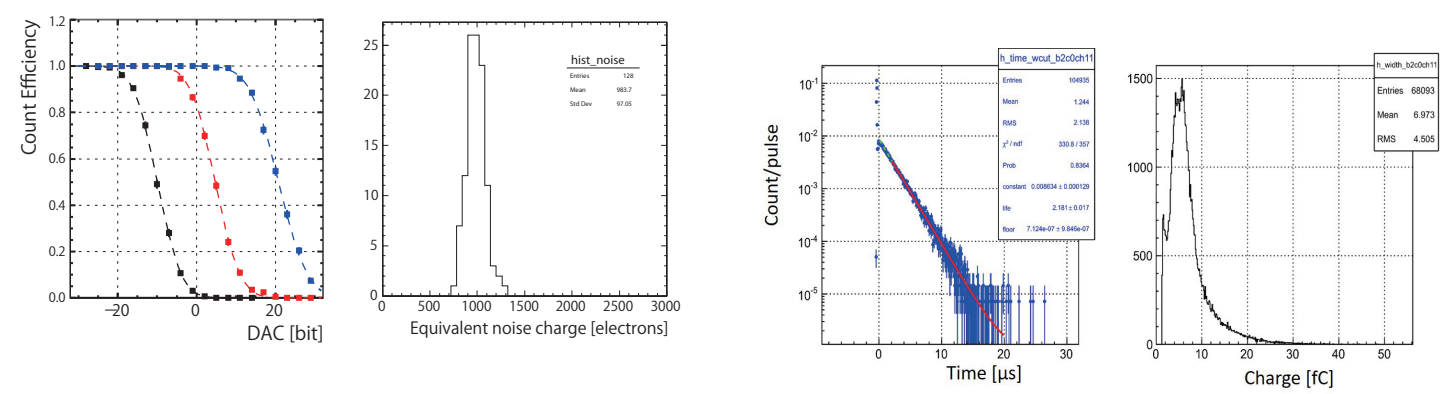

Figure 3: Left: The count efficiency as a function of threshold at the comparator with injected charges of 7,200 (black), 9,600 (red), 12,000 electrons (blue). The data is fitted by an error function. Right: The equivalent noise charge distribution for 128 channels.

Figure 4: Muon decay time distribution (Left) and charge distribution (right). Charge is estimated from the Time-over-Threshold.

The signal from the silicon strip sensor is transmitted through several types of flexible printed circuits (FPCs). The prototype of the FPC for the silicon strip sensor is fabricated by Fujikura Ltd. 
We confirmed the the capacitance of the longest signal line of about $200 \mathrm{~mm}$ is reasonable level $(\sim 13 \mathrm{pF})$ and will start mass production. The designing of other FPCs is ongoing.

We assembled a test module by connecting the full scale prototype ASIC "SliT128A" with a silicon strip sensor thorough signal fan-out flex circuit (i.e., pitch adapters) made by Fujikura Ltd. The assembled test module is tested with a pulsed muon beam at J-PARC. We succeeded in observing positrons from muon decay. Figure 4 shows the measured distributions of muon decay time and charge deposited in the silicon strip sensor.

In parallel to the work on hardware study, we developed software tools for our silicon strip detector. The timing stability is important for the precise measurement of the $(g-2)_{\mu}$. Therefore, we implemented the behaviour of the ASIC into the simulation, and estimated the timing shift due to the pileup. Track reconstruction efficiency should be high and stable against change of hit rate. We developed a track finding tool based on Hough transform method and confirmed the track finding efficiency keeps greater than $90 \%$ even at the expected highest hit rate as shown in Figure 5 .

\section{Conclusion}

We developed the silicon strip detector for the J-PARC muon g-2/EDM experiment. The development of each component for the detector vanes is progressing. We have started to fabricate the silicon strip sensor. The final version of the front-end ASIC "SliT128B" is being finalized. The mass production of the FPCs for the silicon strip sensor will be started. We constructed the test module by combining the full scale prototype ASIC and the silicon strip sensor. We have succeeded in observing positrons from muon decay with a pulsed muon beam at J-PARC. In parallel to work on the hardware study, we developed the track finding tool. According to the simulation study, the track finding efficiency keeps greater than $90 \%$ even at the expected highest hit rate.

\section{References}

[1] G. W. Bennett et al. [Muon g-2 Collaboration], Phys. Rev. D 73, 072003 (2006).

[2] A. Keshavarzi, D. Nomura and T. Teubner, Phys. Rev. D 97, 11, 114025 (2018)

[3] J-PARC E34 experiment web page: http://g-2.kek.jp/portal/index.html

[4] J. Grange et al. [Muon g-2 Collaboration], arXiv:1501.06858.

[5] G. A. Beer et al., PTEP 2014, $091 \mathrm{C} 01$ (2014)

[6] P. Bakule, Y. Matsuda, Y. Miyake, K. Nagamine, M. Iwasaki, Y. Ikedo, K. Shimomura, P. Strasser, and S. Makimura, Nucl. Instrum. Meth-ds Phys. Res., Sect. B 266, 335 (2008).

[7] M. Otani et al., Phys. Rev. Accel. Beams 19, no. 4, 040101 (2016).

[8] H. Iinuma, H. Nakayama, K. Oide, K. i. Sasaki, N. Saito, T. Mibe and M. Abe, Nucl. Instrum. Meth. A 832, 51 (2016).

[9] S. Nishimura et al., JPS Conf. Proc. 8, 025015 (2015).

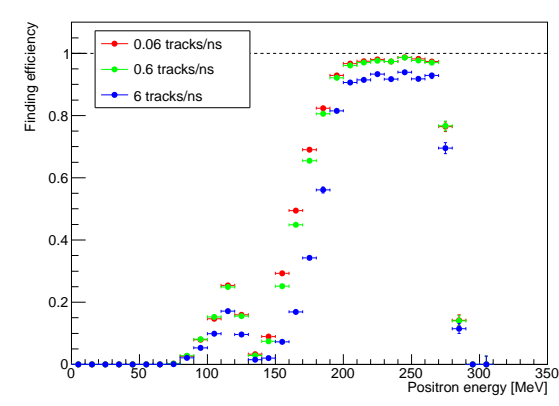

Figure 5: Track finding efficiency as a function of the positron energy with different condition of track rate from 0.06 tracks/ns to 6 tracks/ns. . 